NOTAS Y DISCUSIONES

\title{
Dinámica deliberativa y valores epistémicos ${ }^{1}$
}

\author{
J. FRANCISCO ÁLVAREZ \\ UNED
}

El análisis y la reconsideración de la noción de racionalidad, junto a su posible aplicación en el estudio de la actividad científica, parece una tarea importante para la filosofia de la ciencia. Ese análisis puede ser necesario cuando menos desde una doble vía. La primera aborda el estudio de nuestros modelos ideales de racionalidad, los procedimientos técnicos y formales desde los cuales cabe hablar de una elección racional en el ámbito estricto de la práctica cientifica; una segunda dirección atiende al conjunto de intereses que están actuando en y sobre la práctica cientifica misma $y$, desde otro punto de vista, plantea una consideración "material" de la racionalidad tal como se cxpresa en la práctica científica. Aquí principalmente vamos a comentar algunas lineas de trabajo actuales en la primera dirección; quizá puedan servir como aproximación a determinados problemas epistemológicos que no requieren pasar de inmediato a una consideración "material» y "externan de la ciencia. Tratamos de sugerir una posible consideración de los valores epistémicos como resultado del proceso práctico deliberativo de la misma elaboración científica.

Sin embargo, aunque no haga falta señalar que no es nueva la preocupación por el problema de los diversos valores (morales, cpistémicos, sociales) en la ciencia, recordemos de pasada cómo desde la filosofía de la ciencia se vuelve a plantear esta cuestión en múltiples foros. Por ejemplo, resulta intcresante leer las siguientes observaciones que The Monist propone como orientación para la preparación de su número de abril de 1996, que será un monográfico sobre «Forbidden Knowledge»:

La ciencia no es una actividad libre de valores sino un complejo empeño humano. Ciertas consecuencias de la realización de ese empeño son problemáticas moral o socialmente. Se ha planteado que ese hecho es suficiente para poner en cuestión la libertad de la investigación científica. ¿Hay temas o tópicos que deberian excluirse de la investigación cientifica? Y, si los hay, ¿sobre qué base deberían ser excluidos? La relación del conocimiento científico con otrọs valores, las fuentes y los criterios sobre la restricción de la investigación cientffica pura o aplicada, plantean la cuestión de sí debe darse o no conocimiento prohibido, ${ }^{2}$

No voy a tratar de resolver ese problema aqui, aunque mi opinión es que se da en una situación de elección dificil entre valores en conflicto que exi- 
gen la persistencia en la investigación. No podemos confiar sin más en los resultados de la investigación dejada a un supuesto desarrollo espontáneo, sobre todo porque hay múltiples condicionantes externos de la actividad científica que la ponen al servicio de intereses muy diversos. Aunque puede parecer razonable suponer que por lo menos el apoyo público a ciertos proyectos podría limitarse en situaciones de incertidumbre, o en todo caso no deberían primarse ciertas lineas de investigación, es conveniente reflexionar sobre la práctica científica misma y, al ticmpo que observamos cómo dejada simplemente a su libre desarrollo no parece la ciencia avanzar hacia los mejores resultados sociales, además anotar que ciertos productos internos producen la consolidación de valores epistémicos que pueden actuar como contrastes internos (no todos necesariamente beneficiosos desde el punto de vista social). Me parece que una reflexión sobre el mismo concepto de moionalidad científica y sobre la aplicación de nuestros modelos de racionalidad a la práctica científica podría ponernos en una posible vía de resolución.

Sin embargo la tarea que aqui se propone es más limitada y significa solo un paso para ese análisis más amplio. Haré primero unas observaciones generales sobre lo que podriamos llamar una filosolía escéptica de la ciencia y a continuación veremos cómo los instrumentos analíticos que tenemos para abordar la tarea científica pucden ser un tanto más sofisticados que las habituales comprensiones de la racionalidad cientifica.

Se viene recuperando y ampliando el papel de los elementos discursivos y argumentativos que se dan en la construcción de la ciencia. Además de incorporar como factores causales en la construcción de la ciencia los intereses sociales generales y los intereses propios de los cientílicos, parece conveniente destacar también el papel de los individuos y de sus elecciones racionales en el proceso de toma de decisiones que construye la ciencia. ${ }^{3}$

Los valores que sustentan la investigación no tienen por qué ser un listado de las buenas intenciones y de los rasgos de "olímpica» racionalidad del investigador; antes al contrario; la misma búsqueda de la continuación en el proyecto investigador puede ser una fuente de coherencia interna del proceso y puede producir valores epistémicos intemos a la práctica cientifica. Las decisiones no tienen que verse en una balsa de aceite con bien definidos valores sino en una situación de elección dificil, como ocurre en otros campos de la práctica humana incluyendo amplias zonas de la investigación ética y de la práctica moral. Se pretende asi articular una posición que, si bien puede caracterizarse de perplejidad en la vida moral, puede llamarse escéptica en filosofía de la ciencia.

El ideal ilustrado del poder de la diosa razón aparece secularizadamente como plenamente politeísta; hasta ahora ha sido dominante en filosofia de la ciencia su aparición como la búsqueda y supuesto logro de la verdad definitiva unida a la idea de progreso científico con el correspondiente incremento de la intervención tecnológica en las prácticas sociales. La huida hacia versiones sociologistas como las del denominado programa fuerte, strong program, tiene cierta correspondencia con lo que en otros ámbitos aparecía como el pensamiento débil y, aunque acaso nunca hayamos sido modernos al decir de Bruno Latour, sin embargo, como oposición quizá podríamos definimos como escépticos, una posición epistemológica menos ingenua que la del positivismo o la del relativismo o sociolo- 
gismo científico. Así pues el análisis de la racionalidad científica no debería hacerse hoy desde unos supuestos claramente superados en la discusión epistemológica, ni tampoco a partir de modelos cuya inadecuación ha sido suficientemente mostrada. Indicaremos algunas correcciones internas a los tratamientos formales sobre problemas epistémicos que nos parecen insoslayables para situar en su preciso lugar otros componentes de la práctica científica. Con frecuencia los tratamientos formales resultan inadecuados, pero a veces sus limitaciones no son sólo fruto de la complejidad y variedad de los valores presentes en la ciencia sino que son resultado de nuestra inadecuada modelización.

Por ejemplo, no ha sido infrecuente analizar determinadas formas de razonamiento con nociones procedentes de la teoría de juegos, particularmente ha sido así con el venerable tema de la inducción. Unos primeros intentos no pasaron de vincular el razonamiento inductivo con la búsqueda de una estrategia maximín jugada contra la naturaleza; es decir, el jugador se comporta según su estrategia de seguridad maximizando la mínima ganancia posible. Las conexiones no fueron más allá de relacionar esa estrategia con las diversas nociones de equilibrio en los juegos de estrategia y, en definitiva, se desarrollaron principalmente por vía de la teoria de la inferencia estadística junto a una perspectiva estática o estratégicamente predefinida de los juegos. En esas versiones se discutía el posible equilibrio del juego sin tener en cuenta el proceso de equilibración mediante el cual se supone que se llega al equilibrio. Quizá esa orientación estática hacía de la teoría de juegos un instrumento poco interesante para los problemas epistemológicos $y$, por ello, se produjo cierta reducción de su uso para la reflexion sobre temas epistemológicos.

Ahora bien, ciertos desarrollos posteriores en la teorja de juegos, y la renovación de la discusión epistemológica que se apoya en la teoría de la decisión, permiten pensar temas epistemológicos tradicionales desde una perspectiva procedente de la teoría de juegos. Aquí vamos a indicar exclusivamente algunas líneas del estado de la cuestión y sugerir algunas vías por las que parece discurrir parte de la investigación reciente. En particular, ciertas elaboraciones sobre la propia fundamentación de la teoria de juegos (Ken Binmore), sobre elección dinámica ( $P$. Gärdenfors, I. Levi) y sobre deliberación racional (B. Skyrms), ${ }^{4}$ parecen ofrecer nuevas orfentaciones para abordar la noción de argumentación desde la teoría de juegos y, como elemento derivado, para analizar al menos una nueva forma de hablar, cuando no una nueva perspectiva, sobre temas como el de la inducción, la explicación o la causalidad.

Una adecuada comprensión del proceso de argumentación requiere algo más que observar una conexión entre conjuntos de secuencias de enunciados. ${ }^{5} \mathrm{Si}$ no queremos reducir la argumentación a una presentación sintética de lo que ya es nuestro conocimiento, debemos observar que al argumentar se produce un cambio de nuestra situación cognitiva, de nuestros estados de creencias, de manera que cambian los compromisos que establecemos con nuestro conocimiento. Concluida la argumentación estamos, sea real o pretendidamente, en un estado cognitivo diferente; precisamente en un nuevo estado donde se ha incorporado la conclusión del argumento como parte de nuestro nuevo estado de creencias.

El analisis de la argumentación puede verse enriquecido por las aportacio- 
nes procedentes de los estudios sobre la deliberación racional, la elección en situaciones de conflicto irresuelto y la dinámica de los estados epistémicos. Para el estudio y simulación de la práctica argumentativa interesa ver el rendimiento conjunto de esas tres vas de análisis y observar que en todas ellas se han propuesto diversos modelos de racionalidad que han sido utilizados para estudiar problemas como el de la inducción y el de la explicación científica. Sin embargo, aunque no sea ahora nuestro tema central, la misma noción de racionalidad requiere diversas matizaciones que permitan escapar al simple modelo de la elección racional utilizado principalmente por los economistas y que se muestra muy insatisfactorio. ${ }^{6}$ Esas revisiones, sobre todo el problena de una posible es. tructura de preferencias de segundo orden, pueden también incorporarse en las propuestas de B. Skyrms que veremos más adelante.

Un momento inicial consiste en elaborar modelos que permitan desarrollar ciertos programas de simulación que ofrezcan algunos pasos sobre cómo entendemos la argumentacion. Al abordar la argumentación con la visión tradicional de la teoria de juegos aparecen dificultades si pretendemos entender el juego de la argumentación como un algoritmo en ejecución, sobre todo si consideramos la argumentación como un algoritmo que puede presentar "fallos", como un modelo «imperfecton; estas dificultades se ven fortalecidas por la habitual presentación matricial del juego. ${ }^{3} \mathrm{Si}$ los individuos deben iniciar el debate desde un conocimiento común compartido, referido a su propia racionalidad y creencias, y, si además la racionalidad está constituida exclusivamente por la consistencia de la elección, la ausencia de análisis sobre la génesis de las propias creencias hace que resulte imposible comprender el acceso al equilibrio, salvo que estemos en un universo completamente cerrado. Por tanto, una primera exigencia es intentar modelar el juego de la argumentación en forma extensa, no matricial, de manera que se puedan captar mejor los procesos dinámicos de la argumentación. La presentación extensional pretendería representar las transformaciones de nuestros estados de conocimiento en las diversas fases de la argumentación, mediante lo que podremos llamar transiciones de estado de conocimiento.

Como ha señalado B. Skyrms, epara que la deliberación no sea trivial, debemos mantenernos en una situación de incertidumbre acerca del lugar adonde nos va a conducir la deliberacion. Si sabes adónde vas, ya estás allí. Por tanto, para que la deliberación genere información mediante la computación, el resultado de la computación ha de ser inicialmente incierto" (op. cit., p. 104). Esta posición no parece que se pueda obtener mediante una presentación matricial del juego en la cual los individuos, al suponer que la transformación de sus creencias seguirá el mismo patrón establecido en el momento inicial, adoptan desde el principio una referencia completa sobre cómo serán transformados sus estados futuros.

Sin duda el problema se parece al que afronta cierto bayesianismo ingenuo cuando trata de pasar desde los elementos descriptivos del comportamiento consistente (Savage, De Finetti) a una reinterpretación como teoría normativa (que prescribe, proscribe, señala lo posible y lo permitido). La ingenuidad no se encuentra en el uso de la regla de Bayes (ucuya validez como pieza de álgebra no se cuestiona", K. Binmore, op. cit., p. 142) para la actualizacion del conocimiento - para obtener las 
probabilidades finales a partir de las probabilidades iniciales y de las observaciones realizadas-. La ingenuidad aparece al suponer que se puede aparcar tranquilamente el problema de la conformación de las probabilidades iniciales o las creencias de partida (o algo parecido a los fines y valores que se comparten en el proceso o que se generan en él).

Precisamente la atención a la conformación de las creencias de las que partimos, la reflexión sobre el software que aplicamos y sus posibles transformaciones a medida que avanza el proceso deliberativo nos hace decir que cualesquiera sean los problemas de la inducción científica seguramente están representados por algo más que por la «trivial manipulacion algebraica llamada regla de Bayes» (K. Binmore, ibid., p. 142).

Cuando parece interesante proseguir una investigación y desarrollar una argumentación es cuando no conocemos el resultado. Buscamos algo inesperado que suministrará cierto grado de sorpresa. Ni siquiera en la sistematización deductiva está ausente ese grado de sorpresa de obtención de lo inesperado. Como recuerda Isaac Levi la argumentación surge en un terreno parecido a aquel en que según Dewey se da la necesidad de la teoría moral: es necesaria cuando hay conflicto entre valores ya que si conocemos el bien no tenemos nada que analizar, sólo nos queda practicarlo; cuando aparece un conflicto entre valores es cuando se precisa la investigacion moral.

El conflicto entre nuestro deber moral y la «tentación» presupone que está concluida la investigacion moral sobre lo que es el bien, no albergamos duda sobre lo que debemos hacer; sin embargo suele decirse que los fenómenos relacionados con la debilidad de la voluntad aparecen a partir de cuando sa- bemos qué hacer. No es la debilidad de la voluntad el fundamento de la acción moral; antes al contrario, la teoría moral, la investigación moral, adquiere su sentido más fuerte cuando es anterior a nuestro conocimiento del bien.

Sin duda las situaciones de incertidumbre, a diferencia de las de riesgo calculado, plantean dificultades porque en ellas se generan con suma naturalidad estructuras de "indecision", situaciones de selección diffícil». Por ejemplo, en un marco de incertidumbre radical no podemos asignar a los estados futuros siquiera probabilidad porque desconocemos esos mismos futuros estados, por tanto la elección racional entendida como optimización de alguna función de utilidad no parece en esos dominios ofrecernos guía alguna. Que una teoría, como la de la elección racional, no nos ofrezca una única salida bien definida no es ciertamente un problema si se presenta como teoría normativa porque, posiblemente, en tal caso lo interesante es que nos sugiera líneas de admisibilidad de la acción.

Es precisamente en marcos similares al de esos casos de indecisión de la teoría donde se pueden analizar los conflictos entre las diversas constricciones que sobre las opciones posibles dibujan los diversos compromisos de valor. La imposibilidad del cumplimiento conjunto de todas las ligaduras establecidas por los compromisos de valor, ya sean cognitivos, epistémicos o morales, no señala tanto a la inconsistencia de las creencias del individuo cuanto al punto a partir del cual, tras la suspensión temporal de aquellos valores en conflicto, comienza el proceso de investigación y por tanto de argumentación. Al decir del escepticismo clásico se trata de proseguir en la investigación. $\mathrm{Ni}$ alcanzamos la verdad definitiva como sostiene el dogmático, ni tampoco tenemos que aceptar la im- 
posibilidad de su búsqueda como defendería el relativista radical; por el contrario, tal como sugiere el escéptico es aconsejable seguir en la investigación discrepando de las dos primeras soluciones.

Sin duda parece posible presentar las ideas anteriores a partir de la noción de estados epistémicos de Gärdenfors, pero aquí sólo podremos indicar alguna conexión con sus modelos probabilísticos de los estados epistémicos. Tales modelos se han propuesto en particular para dar razón de la noción de explicacion, aunque como dice Gärdenfors (op. cit., p. 209) el desarrollo de modelos de estados epistémicos más comprensivos y realistas sea un aspecto abierto a la investigación.

Supongamos que $E$ es el explanandum de cierta explicación y apliquemos las ligaduras establecidas por el conjunto de valores cognitivos al estado de conocimiento en el que no se conociese $E$ (contracción del estado de conocimiento a un estado previo); en estas circunstancias, la estrategia de la argumentación consiste precisamente en señalar los mecanismos que incrementan la probabilidad de $E$ a partir de los valores cognitivos -que incluyen un conjunto de creencias y procedimientos, y que por lo tanto establecen la admisibilidad de ciertos conjuntos de situaciones-.

En el estado de conocimiento en el que no se conoce $E$, dado un conjunto de enunciados elementales y enunciados de probabilidad (en el sentido de Gärdenfors), el planteamiento anterior permite introducir una noción de admisión razonable de $E$.

No se trata solo de incorporar la variable temporal para introducir una corecta caracterización de la causa (necesidad sugerida por Gärdenfors en su propuesta de dinámica de los estados epistémicos), ni tampoco parece intere- sante exclusivamente la reducción de las cadenas entre el explanans y el explanandum, opción intentada por otros, sino más bien señalar que el problema consiste en tratar de entender la explicación como cierto equilibrio alcanzado en un proceso de deliberación que se apoya en los procedimientos de argumentación racional. El problema se traslada por tanto a cuáles son las condiciones para considerar racional un estado epistémico y, con ello, la reconsideración de la elección racional vuelve al centro de la cuestión porque aparece como básico estudiar los mecanismos de elección.

No basta estudiar el principio que rige u orienta la eleccion (sea el de la máxima utilidad esperada o cualquier otro) sino el proceso mismo de la deliberación, proceso en cuya ejecución se pueden generar tendencias o sendas de la argumentación a veces como resultado de introducir determinados parámetros de "satisfacción", unos parámetros que se parecerán más a las propuestas tradicionales de la retórica ("contexto", "atmosfera", "convencers) que a las de la lógica. Se trata de modelar individuos que sean capaces de "convencer" a los demás incrementando la probabilidad de $E$ para el estado epistémico del otro, por tanto se trata de organizar una crónica razonable, incorporar en un conjunto adecuado de estado de conocimiento el nuevo dato.

Con lo anterior nos desplazamos hacia una noción de explicación que ya no hereda el problema de la simetría entre explicación y predicción. Corrección necesaria para revisar cierta ingenuidad que aparecía al suponer una simetría preestablecida entre ambas actividades. Este problema de la relación explicacion/prediccion ha supuesto una limitación añadida para muchas formas de teorizacion sobre lo social porque las ciencias sociales también han estado 
muy influidas por la excesiva dependencia de esa simetría explicacion/predicción. Con frecuencia se habla en filosofía de la ciencia de "la estructura de la explicacion", prefiero hablar de "la urdimbre de la explicacions para sugerir algo menos potente que la noción tradicional en filosofía de la ciencia. Cada día se encuentra uno más alejado de quienes ingenuamente pensaban que existfa una interna correlación y casi una simetria entre explicación y predicción. Apoyar cierta ontología a partir de un esquema de explicación ha sido excesivamente frecuente en la mayor parte de las explicaciones en términos de leyes de cobertura de las que siguen más o menos lejanamente el llamado modelo Hempel-Oppenheirn, que en todo caso prefiero llamar milliano (por $\mathrm{J}$. Stuart Mill). Vale la pena recordar una posición como la de Patrick Suppes, quien en 1986, en un artículo titulado "Explicando lo impredictible», con ocasión de un homenaje a Hempel por parte de la revista Erkenntnis, decía:

Se ha dicho - y yo estaba entre quienes lo decian-que cualquier teoría de la expljcación para ser interesante tendría que hacer buenas predicciones. Si no se conseguian esas buenas predicciones la explicación difícilmente se consideraba interesante. Este es otro intento más de unificación que ahora considero equivocado [...] Hemos vuelto a reconocer cuán raros y especiales son los sistemas físicos cuya conducta puede predecirse en detalle. La ingenuidad y las esperanzas de esas épocas tempranas no se sostienen. Para muchos fenómenos en muchos dominios se tienen razones de principio para creer que nunca seremos capaces de movernos de buenas explicaciones a buenas predicciones.

Un paso importante para incorporar la noción de transición durante el proceso mismo de deliberación consiste en entender primero la posibilidad de un tratamiento dinámico de los juegos tal como lo ha desarrollado B. Skyrms, quien sugiere que durante el proceso de simulacion los individuos, que parten de determinadas probabilidades iniciales de elección de una estrategia, se van transformando siguiendo el procedimiento de utilidad esperada según cierto nivel de satisfacción y utilizan los datos que van resultando en el proceso como elementos que se reincorporan al modelo; de tal manera cabe la posibilidad teórica (que se satisface en muchos casos) de obtener una senda de equilibrio y se muestra cómo se accede al equilibrio partiendo de determinadas situaciones de probabilidad de elección de una estrategia que inicialmente eran situaciones muy alejadas del equilibrio.

Este análisis dinámico consigue superar cierto "irracionalismo" que aparece detrás de la definición de equilibrio de Nash. Cuando en un juego que modela cierta situación aparecen varios puntos de equilibrio y no se sabe bien por qué equilibrio optar ni por qué se elige uno determinado, este tipo de juego parece comprometernos en exceso con soluciones «irracionales» o causales. Por ejemplo, en ciertas ocasiones cuando Jon Elster ha analizado juegos que no tienen solución, ${ }^{B}$ juegos que están dados en forma matricial y que tienen varios equilibrios de Nash, parecia sugerir que se necesitaba cierta airracionalidad" para optar por una de las salidas posibles.

Sin embargo, con el tratamiento de Skyms se logra modelar la transición y se refina lo que se puede llamar "dinámica de Nash"; pero, si presentamos un juego en forma extensiva para un proceso de deliberación sin información perfecta, tendremos que incorporar datos sobre la estructura que, durante el mismo proceso de deliberación, exhibirán los nuevos estados epistémicos que van adquiriendo los individuos que delibe- 
ran (en parte en sus versiones más ortodoxas - Selten, 1976- se trata de presentar al agente con su propia «tabla nomal de agente», es decir, se juega in extenso a condición de proponer una versión matricial del agente). La cuestión de introducir esos nuevos inputs surge como una necesidad durante el mismo desarrollo del programa: se necesitan nuevos datos que deben ser estimados e introducidos por otro sujeto que puede ser precisamente el programa modelador de individuos racionales (esta podría ser una lectura de la propuesta de Ken Binmore). Así parece interesante utilizar el trabajo sobre dinámica de la deliberación racional para completar la dinámica de los estados epistémicos. En el fondo cuando se propone una versión probabilitaria de los estados epistémicos su primera modelización podría ofrecerse como una modelización de juego en forma matricial o como una modelización en forma extensa, pero en ningún caso parece requerir la introducción de ligaduras procedentes de determinados compromisos cognitivos. Sin embargo son esos compromisos los que permiten sopesar en cada caso la senda de la deliberación mediante propuestas de técnicas argumentativas - por esta vía se podría intentar utilizar el procedimiento de probabilidades de segundo orden de Skyrms, que sin duda es relevante para nuestro objetivo y que conecta con el tema de las preferencias de orden superior al primero en teoría económica del bienestar.'

En la argumentación parece imprescindible introducir un elemento diná. mico, fluido, que no se detecta en la forma estándar de la explicación. A pesar de su trivialidad es preciso atender a dos cuestiones importantes para el análisis de la argumentación. Por un lado tenemos el punto de partida y el de arribada de la argumentación, pero, por otra parte, debemos fijar tambien nuestra atención, cual viaje a Itaca, en el camino mismo.

La tendencia dominante ha sido la de considerar el discurrir de la argumentacion como un simple mecanismo regulado; sin embargo es muy importante observar la argumentación misma como productora de información para su propio despliegue. La argumentación no es una senda preparada de antemano para que discurramios por ella, no es tanto una trama fija que da estructura a los pasos argumentales cuanto la urdimbre que se va configurando en el proceso mismo de la práctica deliberativa.

Desde luego hay argumentaciones que no admitiremos como explicaciones. Por ejemplo, aquellas argumentaciones que facilitan la aceptación de un resultado y nos comprometen con la crónica sugerida pero que no incrementan la probabilidad de la ocurrencia de ese acontecimiento, no parece que cubran los criterios mínimos para una buena explicación; quizá como candidatas a explicacion tengamos que pensar en algún subconjunto propio del mundo de la argumentación. Qué tipo de argumentaciones se aceptarán como explicaciones es un problema posterior que por ahora no abordaremos, aunque en parte se podría seguir la sugerencia de Gärdenfors, quien considera que estamos ante una explicacion deductiva si suponemos al explanandum $E$ presente también en la ampliación del estado cognitivo que resulta al añadir el explanans al previo estado cognitivo de contracción en el que suponemos la no presencia del explanandum (K. Binmore, op. cit., p. 179). Un resultado bien conocido en el contexto de la teoría neobayesiana de la decisión señala cómo antes de tomar una decisión informada la utilidad esperada es siempre al menos igual a la utilidad de ele- 
gir una decisión no informada $\mathrm{y}$, por otra parte, si hay alguna probabilidad de que la información pueda afectar a nuestra decisión la utilidad de la decisión informada resulta ser estrictamente mayor a la de la decision no informada (resultado de los años cincuenta al que treinta años antes había llegado Ramsey - curiosidad histórica documentada recientemente por Skyrms y N.E. Sahlin), Dado este "valor del conocimiento», en ausencia de algún expediente dinámico "realista" que incorpore los costes de la informacion, tendríamos que suspender la decisión cuanto supusiésemos una estrategia de retroalimentación informativa: si siempre pudiesemos disponer de información libre de costes y si pudiésemos retrasar la decisión nunca llegaríamos a adoptarla, estariamos siempre a la espera de obtener algún nuevo tipo de información. Así, la formulación clésica del diálogo que queda abierto a la discusion y en el que siempre se sigue a la espera de nuevas e inesperadas informaciones resulta coherente, porque no hay limitaciones de tiempo, pero toda pretensión de obtener una solución definitiva sería falaz.

Sin embargo, si lo que pretendemos es analizar un tipo de argumentación con la que se pretende hacer plausible cierta opción de conocimiento, debemos reconocer algo cognitivamente inevitable: partimos de la aceptación de cierto estado de conocimiento $y$ de la configuración de un determinado modelo de agente argumentador; a partir de ahí, y utilizando procedimientos argumentativos disponibles en la configuración del agente, se van aceptando determinadas conclusiones que se incorporan al conocimiento compartido y que en algún caso deben pasar por la misma contrastación del modelo del agente. Se trata de pensar en situaciones en las que no se puede prescindir, al menos como punto de referencia último, del individuo como soporte de esos procesos cognitivos; si esto suena a escepticismo irracionalista al tratar el tema de la inducción nos parece que con Hume no estamos en mala compañfa, a pesar de las críticas de Russell sobre este mismo tema, y sólo nos cabe decir que se trata de incorporar al individuo concreto, sea individuo animal humano o computador, y que, en todos los casos esa individualidad aparece como un inevitable parámetro que sustenta el proceso cognitivo, que sobre todo es fundamental si queremos pensar en los errores y en lo inesperado. Estamos hablando pues de individualismo cognitivo, aunque pueda ser en muchos casos un elemento repetible que corresponda a diversos tipos de agente y que tenga componentes de aprendizaje social.

Interesa scñalar que no nos proponemos resolver el problema de la explicación inductiva aunque sólo sea porque «La inducción no la vamos a entender como un método de inferencia hacia la mejor explicación sino a la mejor sistematización». ${ }^{10}$ Esa mejor sistematización en nuestro caso tiene mucho que ver con una forma particular de observar la argumentación $y$, por supuesto, con lo que en ocasiones se ha llamado lógica del descubrimiento en la que posiblemente tengamos que atender al «juego retórico» porque, en definitiva, se trata de llegar a situaciones de equilibrio cognoscitivo en las que encontremos cierto acuerdo y estabilidad. No buscar la optimización de los procesos sino analizar su dinámica particular exige superar una noción formalista de la argumentación e incorporar la consideración pragmática sin perder por ello el objetivo de la "máquina de hacer argumentos".

Tratamos de argumentar y razonar sobre dominios concretós y esto exige 
de nosotros que hagamos constantes simplificaciones. Nos encontramos persistentemente con la necesidad de dejar sin conocer muchos hechos, no decirlos o resumirlos, condensarlos. Como ha dicho J. Pearl: wel arte de razonar bajo incertidumbre equivale al de representar y procesar resúmenes de excepciones». Es interesante tener presente que en inteligencia artificial los sistemas expertos de primera generación han trabajado con las excepciones, asignando a las proposiciones medidas numéricas que se combinaban de acuerdo a principios de uniformidad sintăctica a la manera como los valores de verdad se combinaban en logica, pero con ello se han producido resultados muy extraños y contraintuitivos. El asunto consiste en que las medidas de la incertidumbre se proponen para algo muy diferente de para lo que proponemos los valores de verdad. Debemos tener en cuenta, como dice Pearl, que si los valores de verdad en lógica caracterizan a las fórmulas que discutimos, las medidas de incertidumbre pretenden caracterizar las excepciones, es decir, los hechos que no se muestran en las formulas. aMientras la sintaxis de las formulas es una guía perfecta para la combinación de los visibles, es casi inútil para combinar los invisibles [...] Tales excepciones invisibles pueden interactuar de intrincados $y$ ocultos modos, como resultado perdemos la mayor parte de los rasgos computacionales atractivos de la lógica clásica: su modularidad y el carácter monotónico.» Justamente uno de los aspectos relevantes del tratamiento de las estructuras de valor por parte de I. Levi, que ha sido aprovechado en esta orientación por Gärdenfors, es la posibilidad de un tratamiento de los resultados no monotónicos, por ejemplo, los resultados que aparecen en el cambio del "orden" de la inferencia.
Estas ideas generales pretenden indicar algunos caminos para desarrollar programas de análisis de la trayectoria de deliberación racional simulando juegos muy simples del tipo que ha propuesto Skyrms en The Dynamics of $R a-$ tional Deliberation. Esos programas con toda su sencillez resultan muy sugerentes ya que el tratamiento dinámico y la presentación extensiva de los juegos indica nuevas perspectivas para ol análisis de temas epistemológicos tradicionales.

Es muy probable que con los valores epistémicos nos encontremos con componentes de la práctica científica que juegan papel análogo a los sentimientos primarios en psicología. Así es posible que la memoria y la anticipación jueguen un papel decisivo a la hora de establecer el tipo de valor epistémico que tenemos en cuenta. Es probable que haya pasado el momento de utilizar las metáforas estáticas que siguen dominando la filosofía y particularmente la contemporánea filosofía de la ciencia. Parece oportuno dedicar mayor atención a las prácticas argumentativas $y$ a las discusiones que se producen fuera del estricto ámbito de la disciplina, atendiendo a similar preocupación que la manifestada por algunos economistas con relación a sus modelos de ser humano pretendidamente racional que se restringe a la racionalidad instrumental, y, por el contratio, atender a la posibilidad de elaborar modelos que traten de representar cierta ediscusión racional» sobre los fines con todo lo fragmentaria, dinámica e incompleta que sea. Hay quienes han sugerido que es hora de libar en otras fuentes para nuestras metáforas, quizá la Química, ciertas obras literarias, los modelos cristalográficos o la Topología sean buenos lugares para acercarnos algo más a versiones dinámicas." 


\section{NOTAS}

1. Un primer avance de este trabajo se presentó como comunicación al Encuentro de Logica y Filo. sofia de la Ciencia: Cantap, Reichenbach in meno. riam (Madrid, 13-15 de noviembre de 1991). Posteriomente, con un mayor deslizamiento favorable hacia las versiones escepticas, he podido discttir algunos aspectos en el encuentro Ciencia en perspective (Salamanca, 11-13 de mayo de 1995). organizado por Femando Broncano. Debo agradecer el apoyo de la DGICYT que me permitió pasar un semestre del año 1994 en el Darwin College de Cambridge rabajando con el profesor Geoffrey Lloyd sobre estos y otros asuntos en historia de la ciencia antigua. También ha sido de gran ayuda para este trabajo mi vinculacion al proyecto de la DGICYT PS 92-0031.

2. Información obtenida via Internet utilizando el Gopher de la American Philosophical Association.

3. Resulta muy provechosa la lectura de una muy adecuada presentación de los dos polos y sus tensiones como aparece en $\mathbf{C}$. Solis: Razones e intereses. La historia de la ciencia después de Kuhn, Bamelona, Paidos, 1994

4. K. Binmore, Essays on the Foundations of Game Theory, Oxford, Basil Blackwell Ltd., 1990. B. Skyrms, The Dymamics of Rational Deltberation, Cambridge, MA, Ilarvard U. Press, 1990. P. Gärdenfors, Kitowledge in Flux, 1987. I, Levi, Hard Chotces. Decision makitg under untesolved conflict, Cambridge, Cambridge U. Press, 1986, 1990.

5. Observación planteada en diversas ocasio. nes por Eduardo Bustos, con quien he podido discutir en diversas ocasiones las múltiples consecuencias de esta aparentemente simple pro. puesta.

6. Algunas de esas matizaciones, principalmente las derivadas de los análisis de A. Sen, por ejemplo, en «Rational Fools* las he tenido en cuenta en ${ }_{C}$ Es inteligente ser racional? $*$, Sistema, 109, pp. 73-91.

7. Se presenta como punto de partida la es tructura de preferencias de los participantes y una tabla que nos indica las estrategias de los agentes. El modolo del juego aparece de antemano, ya sabemos previamente nuestra mejor estrategia con independencia del camino seguido por el juego.

8. Estudios aparecidos en múltíples lugares. Baste recordar dos de sus primeros libros en los que aparecia este problema: Loyic and Society (1978). Explaining Technical Change (1983). Se pueden encontrar versiones espanolas algo « trañas*.

9. Ampliación de la noción de preferencias que es frecuente unir al nombre de Amartya $K$. Sen.

10. N. Rescher, Induction. An essay on the justiftcation of inductive reasoning, Pittsburgh, $\mathrm{U}$. of Pittsburgh Press, 1980.

11. He tenido la suerte de discutir estos temas con muchas y muchos colegas preocupados por el tema de las metáloras. Desde luego Jose Gon* zalez, Ana Sanchez, Maria Herrera, Carlos Thiebaut, Mariflor Aguilar y Eduardo Bustos están conmigo en ese dinámico juego. 